\title{
Prevalence of Yersinia enterocolitica in Locally Produced and Imported Veal Mincemeat in Baghdad
}

\author{
Ali H. A. Al-Shammary ${ }^{1}$, Ibrahim A. H. Al-Zubaidy ${ }^{2}$, Methaq G. Abd Al-Rubaey ${ }^{3}$ \\ Zoonoses Diseases Research Unit/Veterinary College/Baghdad University
}

\begin{abstract}
In order to investigate the prevalence of Yersinia enterocolitica in vealmincemeat, ninety pooled samples (three replicates from each type, $n=270$ ) were randomly collected from different markets, butcher shops, free sellers and vendor grills in Baghdad during April until December (2015), in which they processed and analyzed by different food microbiological procedures. The results showed isolation and identification of 71 (78.9\%) isolates out of 90 pooled samples as $31(34.45 \%)$ isolates from 45 locally produced veal mincemeat: 7 (15.55\%) isolates from 10 butcher shops mincemeat, $12(26.67 \%)$ isolates from 20 free sellers' mincemeat and 12 (26.67\%) isolates from 15 vendor grills; and 40 (44.45\%) isolates from 45 imported veal meat (minced locally for human consumption): 27 (30\%) isolates from 35 free sellers and 13 (14.45\%) isolates from 10 vendor grills.The mean log count of Yersinia enterocolitica in locally produced mincemeat range from 3.176 to $4.230 \log _{10} \mathrm{cfu} \mathrm{g}^{-1}$, while in imported ones range from 6.740 to $9.826 \log _{10} \mathrm{cfu} \mathrm{g}^{-1}$. Y. enterocolitica-like bacteria were evident and detected in most samples. Results profile provide useful information on biosafety and hazard analyses critical control points of hygienic measurements of vealmincemeat marketed in Baghdad
\end{abstract}

Keywords: Yersinia enterocolitica, Veal Mincemeat.

\section{Introduction}

Yersinia enterocolitica was discovered by Schleifstein and Coleman in 1939.Y. enterocolitica was known as a psychrotrophic waterborne and foodborne enteropathogen. Outbreaks of yersiniosis were commonly associated with food vehicles such as meat (particularly pork), milk, powdered milk, cheese, tofu and raw vegetables. The first and definitive food associated outbreak of yersiniosis occurred in Oneida County, New York, where over 220 individuals (primarily school-age children) were stricken with acute gastroenteritis after the consumption of contaminated milk. Y. enterocolitica has been isolated from meat, chicken, vacuum packaged meat, pork, ham, drinking water, milk and oysters. pathogenic $Y$. enterocolitica synthesizes and secretes several outer membrane proteins (Yops), which play a major role in virulence (1-5).

Y. enterocoliticawas a zoonotic agent that causes gastrointestinal disease in humans, as well as reactive arthritis and erythema nodosum. Enteropathogenic strains were the etiological agents for yersiniosis, which can be acquired through the consumption of contaminated foods. As porcine were the main carriers of $Y$. enterocolitica, food safety measures to minimize human infection were of increasing interest to the scientific and medical community. The frequency of isolation of $Y$. enterocolitica during recent years has increased dramatically, causing much concern.During the past 10 years increasing evidence has accumulated that $Y$. enterocolitica infections were very frequent in some parts of the world, and the infection was probably common but unrecognized in many other countries. The increase in reported isolations was probably a result of greater awareness about this organism and about its potential role in human and animal disease (6-10).

$Y$. enterocolitica and $Y$. enterocolitica-like bacteria constitute a fairly heterogeneous group of bacteria which both wellestablished pathogens and a range of environmental strains which are ubiquitous in terrestrial and freshwater ecosystems. Pathogenic significance in man was mainly associated with a few serogroups (O:3, O:9, O:8, O:5,27). The pathogenic serogroups show different geographical distributions. The development of isolation procedures which clearly differentiate pathogenic from non-pathogenic variants has been difficult. Of special significance in food hygiene was the ability of $Y$. enterocolitica to grow in refrigerated foods.Improved isolation methods and DNA colony hybridization using genetic probes has indicated that the prevalence of pathogenic $Y$. enterocolitica in meat products was substantially higher than previously suggested. Prevention and control measures should focus on information of people involved in food processing and preparation and on the improvement of hygiene during slaughtering of meat producing animals (11-13).

The incidence of $Y$. enterocolitica foodborne infection varies according to geography and climate variation. In developed countries, the incidence is higher in infants and young children, although all ages were at risk. The majority of food borne infections were sporadic, and the infection sources were unknown, but large outbreaks have also occurred (8). Virulence in $Y$. enterocolitica was mediated by both chromosomal and plasmid-borne genes. While chromosomal determinants were stable, plasmids containing virulence genes may be lost during culture and conformational procedures (14).

Currently, there was limited information regarding the prevalence of $Y$. enterocolitica and $Y$. enterocolitica-like bacteria in mincemeat in Iraq. Therefore, this study was conducted to determine the contamination ratio of $Y$. enterocolitica and $Y$. enterocolitica-like bacteria from locally and imported retail raw veal mincemeat in Baghdad markets.

\section{Materials and Methods}

Collection and Processing of Samples: a total of ninety pooled samples (three replicates from each type, totally: two hundred seventy replicates) were randomly collected from 


\section{International Journal of Science and Research (IJSR) \\ ISSN (Online): 2319-7064 \\ Index Copernicus Value (2013): 6.14 | Impact Factor (2015): 6.391}

different markets, butcher shops, free sellers and vendor grills in Baghdad during April until December (2015), in which they processed and analyzed by different food microbiological procedures with some modifications. Samples were collected aseptically in sterile plastic bags and containers, in which they transported to zoonotic lab as soon as possible (14-24).

Numerous enrichment schemes have been described for the recovery of $Y$. enterocolitica from meat samples. These enrichment procedures include cold enrichment for up to a month, direct selective enrichment, or two-step preenrichment/selective enrichment procedures. It appears that some enrichment procedures were better suited for the recovery of pathogenic $Y$. enterocolitica than others, though recovery may be influenced by the type of meat product. Even when using an enrichment and plating scheme reported to give good recovery from a particular meat product, considerable variation in recovery may be observed. Methods reported to provide good recovery of pathogenic $Y$. enterocolitica in one part of the world may not work so well in another geographical area, possibly due to differences in levels of $Y$. enterocolitica and competing flora as well as, animal species and type of products, i.e. mincemeat may harbor more microbial log than whole meat possibly due to redistribution of Yersinia (14).

Each pooled and well mixed replicates were divided in to two separate units (direct and indirect processing): directly well mixed replicates units were enriched on freshly prepared tryptone soya yeast extract broth for 24-72 hours at $4,10,25 \& 37{ }^{\circ} \mathrm{C}$ (50 g well mixed sample part was added to 250-500 ml tryptone soya yeast extract broth, then mixed well by vortex and streaked by sterile loops and swabs on selective chromogenic Yersinia CIN (cefsulodin-irgasannovobiocin) agar plates (Oxoid UK) according to the method of the FDA for each pooled unit), then incubated at $37{ }^{\circ} \mathrm{C}$ for 18-48 hours. In this pathway samples units were cultured by dilution formula: one pooled part of sample unit to five-ten parts of broth diluent (14-16). Indirectly pooled replicates were refrigerated at $4{ }^{\circ} \mathrm{C}$ for 3-7 days then either enriched with tryptone soya yeast extract broth or diluted with sterile phosphate buffered saline (one-unit part of sample to ten parts of diluent) at $10,25 \& 37{ }^{\circ} \mathrm{C}$ for $3,7,10 \& 14$ days, then sub cultured on CIN agars at same temperatures and incubation periods above (14-24).

Pure isolated pink bull eyes colonies with clear watery borders were counted by droplet technique in accordance to McFarland's opacity tubes, then pure seeds were prepared for further identification procedures. Electronic computerized biochemical RapID ${ }^{\mathrm{TM}}$ One panel test system for Enterobacteriaceae was used for confirmation procedure of isolates.All the isolates which were negative for utilization of citrate, positive for urease activity and giving an alkaline slant/acid butt without gas or $\mathrm{H} 2 \mathrm{~S}$ onKligler iron agar were submitted to further testing. In order to identification and bio grouping of isolates as $Y$. enterocolitica; activities of oxidase, lysine decarboxylase, ornithine decarboxylase, $\beta$-Dglucosidase, lipase and pyrazinamidase, utilization of rhamnose, sucrose, xylose and trehalose were evaluated. Further analyses were also conducted applying Indole and Voges Proskauer tests to isolates (14-24).
Testing for Pathogenicity Markers: $Y$. enterocolitica strains were tested for virulence by Temp-Dependent autoagglutination $\left(25^{\circ} \mathrm{C}-35^{\circ} \mathrm{C}\right)$ in Methyl Red-Voges Proskauer broth, occur of small red colonies on Congo RedMagnesium Oxalate agar and Congo red /crystal violet binding assays (calcium binding and biofilm formation) as well as, esculin hydrolysis, fermentation of salicine and formation of formazan red tree on modified 2,3,5tritetrazoliūm chloride semisolid nutrient agar tubes. CIN (without selective supplements), MacConkey, SorbitolMacConkey, Salmonella-Shigella and Eosin Methylene Blue agars were used with Enterobacteriaceae biochemical panel test for identification of $Y$. enterocolitica-like bacteria (1424). Data were statistically analyzed by Chi-square test in accordance with SPSS (25).

\section{Results \& Discussion}

Results profile reflect contamination of mincemeat samples with $Y$. enterocolitica and $Y$. enterocolitica-like bacteria in Baghdad as shown in tables (1, $2 \& 3)$ and photographs (1). The results showed isolation and identification of $71(78.9 \%)$ isolates out of 90 pooled samples as 31 (34.45\%) isolates from 45 locally produced veal mincemeat: 7 (15.55\%) isolates from 10 butcher shops mincemeat, 12 (26.67\%) isolates from 20 free sellers' mincemeat and 12 (26.67\%) isolates from 15 vendor grills; and 40 (44.45\%) isolates from 45 imported veal meat (minced locally for human consumption): 27 (30\%) isolates from 35 free sellers and 13 $(14.45 \%)$ isolates from 10 vendor grills. The mean log count of $Y$. enterocolitica in locally produced mincemeat range from 3.176 to $4.230 \log _{10} \mathrm{cfu}^{-}{ }^{-1}$, while in imported ones range from 6.740 to $9.826 \log _{10} \mathrm{cfu} \mathrm{g}^{-1}$. Y. enterocolitica-like bacteria were recovered and detected in most samples. Results profile provide useful information on biosafety and hazard analyses critical control points of hygienic measurements and contamination ratio of veal mincemeat marketed in Baghdad.

Table 1: Isolation percentages and mean $\log _{10}$ count of $Y$. enterocoliticafrom locally produced vealmincemeat in

Baghdad

\begin{tabular}{|c|c|c|c|}
\hline $\begin{array}{c}\text { Type of } \\
\text { Mincemeat }\end{array}$ & Number & $\begin{array}{c}\text { Isolation } \\
\%\end{array}$ & $\begin{array}{c}\text { Mean } \log _{10} \\
\text { count cfu } \backslash g^{-1}\end{array}$ \\
\hline Butcher Shops & $10(\mathrm{r} 30)$ & $7(15.55 \%)$ & $4.230^{\mathbf{a}}$ \\
\hline Free Sellers & $20(\mathrm{r} 60)$ & $12(26.67 \%)$ & $4.230^{\mathbf{a}}$ \\
\hline Vendor Grills & $15(\mathrm{r} 45)$ & $12(26.67 \%)$ & $3.176^{\mathbf{b}}$ \\
\hline Total & $45(\operatorname{tr} 135)$ & $31(34.45 \%)$ & 3.703 \\
\hline
\end{tabular}

a,b: Indicate significant differences among isolates for mean $\log _{10}$ count vertically at level $(\mathrm{P} \leq 0.05)$.

Table 2: Isolation percentages and mean $\log _{10}$ count of $Y$. enterocolitica from imported veal meat minced locally in Baghdad

\begin{tabular}{|c|c|c|c|}
\hline Type of Mincemeat & Number & Isolation \% & 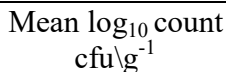 \\
\hline Free Sellers & $35(\mathrm{r} 105)$ & $27(30 \%)$ & $9.826^{\mathrm{a}}$ \\
\hline Vendor Grills & $10(\mathrm{r} 30)$ & $13(14.45 \%)$ & $6.740^{b}$ \\
\hline Total & $45(\operatorname{tr} 135)$ & $40(44.45 \%)$ & 8.283 \\
\hline
\end{tabular}

a,b: Indicate significant differences among isolates for mean $\log _{10}$ count vertically at level $(\mathrm{P} \leq 0.05)$. 


\section{International Journal of Science and Research (IJSR) ISSN (Online): 2319-7064 \\ Index Copernicus Value (2013): 6.14 | Impact Factor (2015): 6.391}

Presumptive isolation and identification of $Y$. enterocoliticalike bacteria from veal mincemeat in Baghdad were checked by selective and differential media with biochemical panel test and Oxoid serotyping kit for E. coli O157 H7.

Table 3: Isolation percentages of $Y$. enterocolitica-like bacteria from veal mincemeat in Baghdad

\begin{tabular}{|c|c|c|c|}
\hline Type of Isolate & $\begin{array}{c}\text { Locally } \\
\text { produced } \\
\text { Mincemeat }\end{array}$ & $\begin{array}{c}\text { Imported } \\
\text { Mincemeat }\end{array}$ & Total \\
\hline Escherichia coli & $35(77.8 \%)$ & $42(93.4 \%)$ & $74(82.3 \%)$ \\
\hline E. coli O157 H7 & $17(37.8 \%)$ & $27(60 \%)$ & $44(48.9 \%)$ \\
\hline Klebsiella pneumoniae & $3(6.7 \%)$ & $2(4.5 \%)$ & $5(5.6 \%)$ \\
\hline Enterobacter aerogenes & $2(4.5 \%)$ & $3(6.7 \%)$ & $5(5.6 \%)$ \\
\hline Enterococcus faecalis & $1(2.3 \%)$ & $1(2.3 \%)$ & $2(2.3 \%)$ \\
\hline Salmonella enterica & $7(15.6 \%)$ & $11(24.5 \%)$ & $18(20 \%)$ \\
\hline Pseudomonasaeruginosa & $9(20 \%)$ & $12(26.7 \%)$ & $21(23.4 \%)$ \\
\hline Proteus mirabilis & $4(8.9 \%)$ & $4(8.9 \%)$ & $8(8.9 \%)$ \\
\hline Aeromonashydrophila & $1(2.3 \%)$ & $3(6.7 \%)$ & $4(4.5 \%)$ \\
\hline Citrobacter freundii & $1(2.3 \%)$ & $1(2.3 \%)$ & $2(2.3 \%)$ \\
\hline Serratia marcescens & $1(2.3 \%)$ & $1(2.3 \%)$ & $2(2.3 \%)$ \\
\hline
\end{tabular}

CIN (Cefsulodin, Irgasan, Novobiocin) agar was a highly selective medium designed to isolate Yersinia enterocolitica. The properties of this medium were based on selective chemical agents, antibiotics, dyes, and the basal medium. Its highly selective against the growth of Escherichia coli, Klebsiella pneumoniae, Proteus mirabilis, Pseudomonas aeruginosa, Salmonella enterica, Shigella sonnei and Streptococcus faecalis. The characteristic deep red center with a transparent margin, or "bull's-eye" appearance of Yersinia and Aeromonas colonies was important for identification, and was due to the presence of mannitol. $Y$. enterocolitica ferments the mannitol in the medium, producing an acid $\mathrm{pH}$, which gives the colonies their red color and the "bull's eye" appearance. Sodium deoxycholate, cefsulodin, irgasan, and novobiocin were added as selective agents. Colonies of $Y$. enterocolitica appeared small, medium and large mucoid with convex and shiny dew drop texture as red centered bull eyes with transparent borders, while similar colonies of Aeromonashydrophila had entire center and borders red texture. Colonies of $Y$. enterocolitica have very offensive odour with characteristic pink pigmentation as clouds surrounding bull eyes colonies on CIN medium. Highly selective biochemical RapID ${ }^{\mathrm{TM}}$ One panel test kit (Oxoid, 2015) was used for confirmation of $Y$. enterocolitica and $Y$. enterocolitica-like bacteria with diagnostic microcodes revealed and confirmed contamination levels in mincemeat in Baghdad. Characteristic features of Yersinia colonies on MacConkey agar were tested as pale, large, mucoid, glistening teeth like structures. Biofilm producing strains were detected in most isolates by Christensen microtiter plate assay and Freeman Congo Red agar, then tested for antibiotic resistancy in another field $(1,14)$.
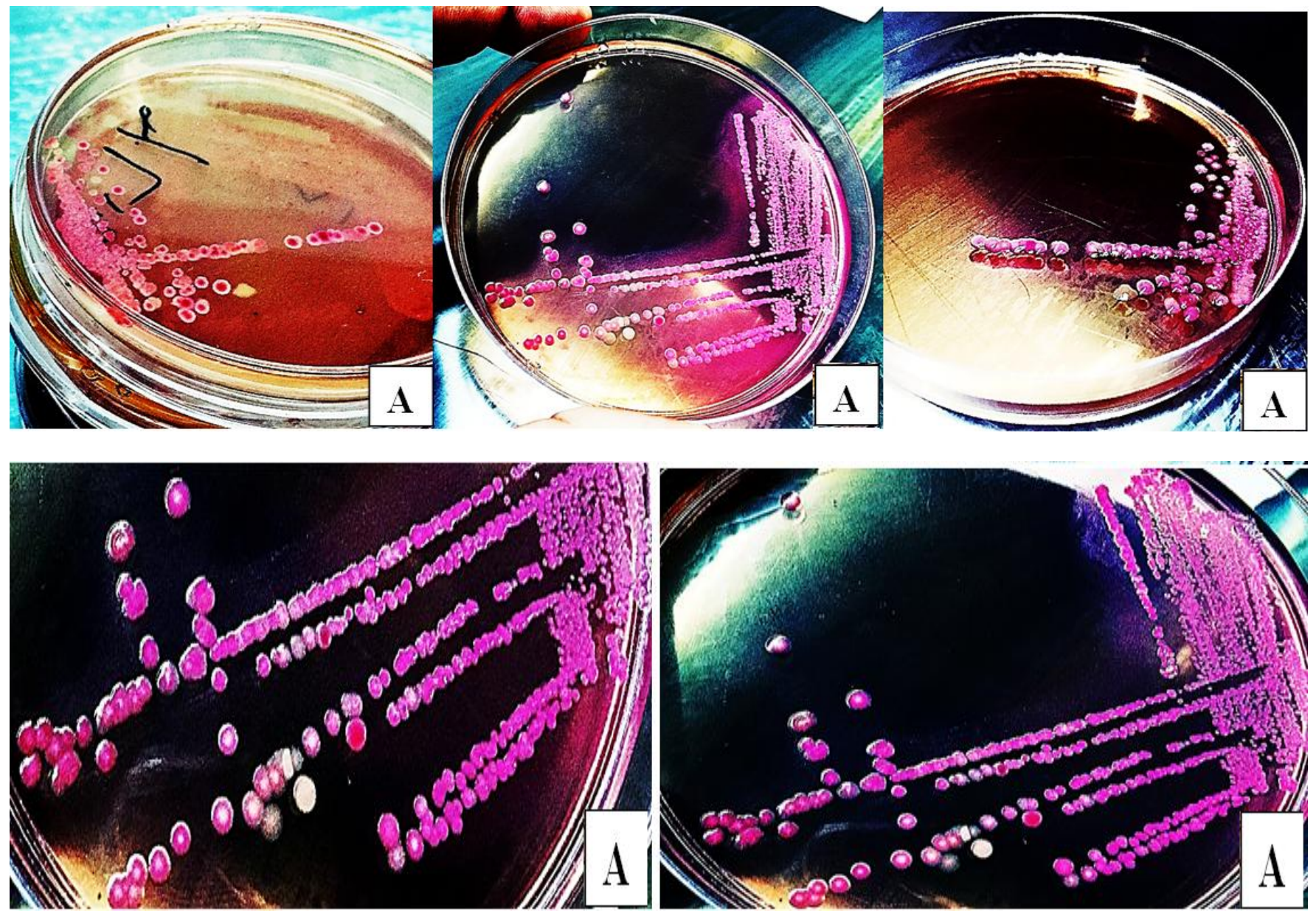


\section{International Journal of Science and Research (IJSR) \\ ISSN (Online): 2319-7064}

Index Copernicus Value (2013): 6.14 | Impact Factor (2015): 6.391

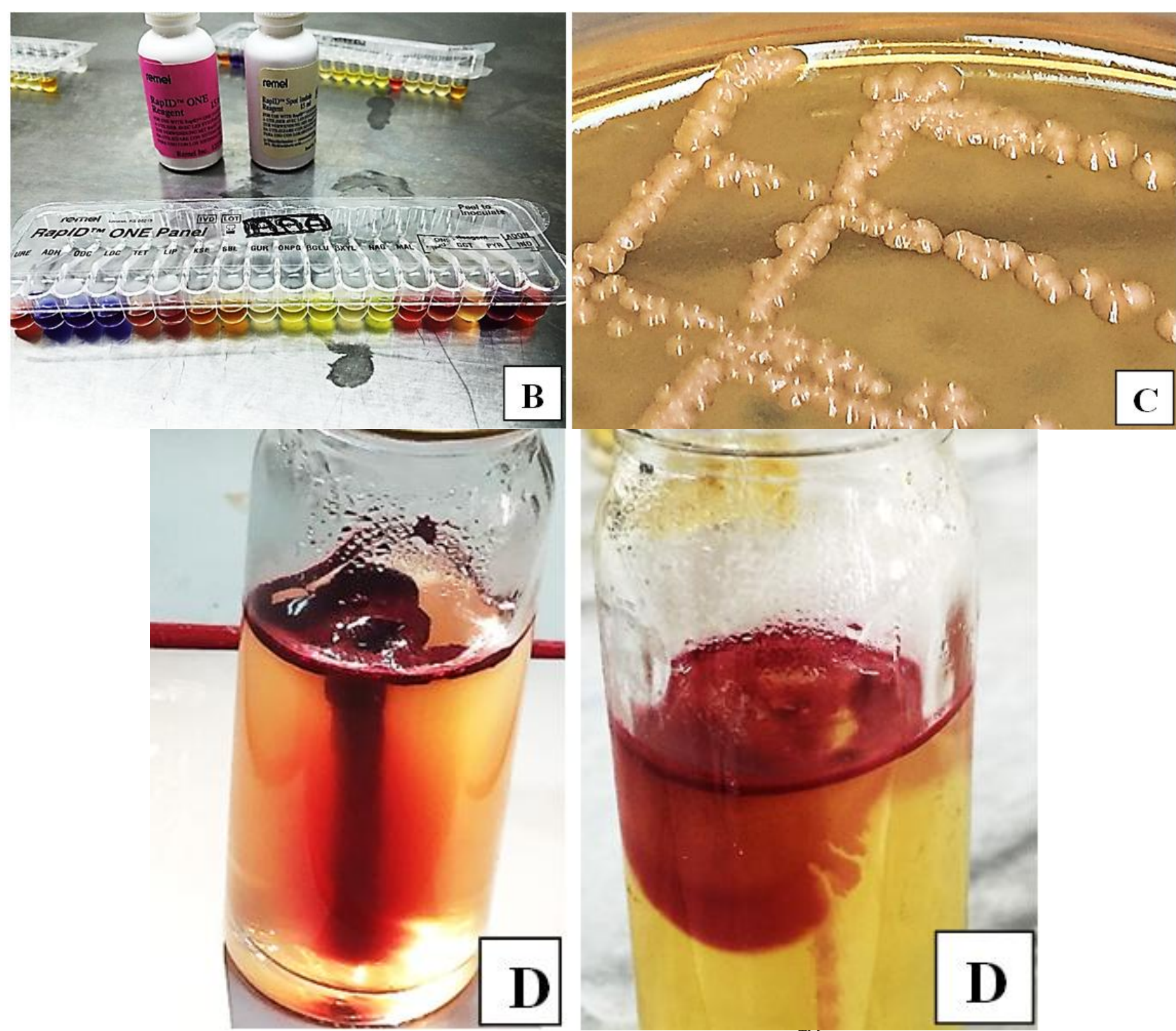

Photographs 1: $Y$. enterocolitica on CIN agar (A), MacConkey agar (B), RapID ${ }^{\mathrm{TM}}$ One Panel (C) and modified TTC semisolid medium (D)

E. coli and their pathogenic serotypes $O 157 \mathrm{H} 7$ were recovered from most samples as a major contaminant pathogen even in cold stages of isolation; this may indicate strong relationship between these isolates in mincemeat.

The serotyping and biotyping of isolates within $Y$. enterocolitica species can be helpful in determining whether they were potential pathogens. In the absence of the antisera to serogroup $Y$. enterocolitica isolates in routine microbiology laboratories, significance remains a function of assessing an isolate for plasmid-encoded virulence factors. These tests were indirect but simple markers of pathogenicity that can be determined in most laboratories and include autoagglutination, production of $\mathrm{V}$ (immunogenic protein) and $\mathrm{W}$ antigens (nonprotective lipoprotein), serum resistance, calcium dependency for growth at $37{ }^{\circ} \mathrm{C}$, Congo red and crystal violet binding tests, and even plasmid profiles. Other virulence assays include lethality for mice, production of conjunctivitis in guinea pigs (Sereny test), absence of pyrazinamidase activity, hydrolysis of esculin $\left(25^{\circ} \mathrm{C}\right)$, and fermentation of salicin $\left(35^{\circ} \mathrm{C}\right)(1)$.
Recovery of pathogenic $Y$. enterocolitica was contingent upon a number of factors including: the level of background flora on the product; the amount of background flora coming through enrichment and plating; the level of pathogenic $Y$. enterocolitica present on the sample; the numbers of nonpathogenic $Y$. enterocolitica and non-pathogenic Yersinia spp. present on the product; and loss of virulence factors during enrichment and plating. Furthermore, a recovery method which gives good recovery of one serotype of pathogenic $Y$. enterocolitica may not be suited to other serotypes. In order to recover any of the important pathogenic serotypes of $Y$. enterocolitica which might be present, multiple enrichment broths and plating media are usually recommended for the recovery of the organism from naturally-contaminated foods.

A great deal of effort must be expended in the recovery and characterization of presumptively-pathogenic $Y$. enterocolitica. Sequential levels of characterization tests include: identification of presumptive Yersinia, speciation to $Y$. enterocolitica, biogrouping the $Y$. enterocolitica, followed by testing for pathogenicity markers. $Y$. enterocoliticawas

\section{Volume 5 Issue 4, April 2016}




\section{International Journal of Science and Research (IJSR) \\ ISSN (Online): 2319-7064}

Index Copernicus Value (2013): 6.14 | Impact Factor (2015): 6.391

more active biochemically at $25^{\circ} \mathrm{C}$ than at $35-37^{\circ} \mathrm{C}$, meaning that disparate results for a given test may be obtained depending onincubation temperature.

These findings suggest presence of complex scenarios of contamination cycle with $Y$. enterocolitica and $Y$. enterocolitica-like bacteria from locally produced and imported retail raw veal mincemeat in Baghdad markets; contamination may occur from farm to markets, during unhygienic free selling, vendor grilling of unknown source of mincemeat and during shipment of stressed and infected animals, inside abattoirs during slaughtering and evisceration and during handling and processing of meat by infected and carrier workers, knives, butcher cutting wood surface, mincing machines, contaminated utensils, flies, unclean meat environment, etc.

As we know, most slaughtering today in Baghdad performed outside abattoirs with unhygienic monitoring system, so that repeated cycles of contamination and pollution occurs in presence of different transmitters and vehicles particularly flies leading to production of dirty, unclean and unhealthy meat and meat products as well as the unacceptable ethics in meat processing and handling with unclean environment, measurements and practices inside most butcher shops with open retailing of these mincemeats in unclean and contaminated containers in Baghdad markets. The differences between the findings of various authors and those of this study might be due to several factors such as isolation methods, number of analyzed samples, season, and geographical location. These factors may cause an increase or decrease in the prevalence of the Yersinia spp. For instance, the present study was carried out in Baghdad province, where the climate is generally warm and humidity is high. It is known that the isolation ratio of $Y$. enterocolitica is higher in colder climates.

Unrestricted hygienic monitoring systems and food policies like absence of bio safety and hazard analysis critical control points during production and handling of healthy meat, absence of risk assessments during importation of meat and meat products, all these and others result in contamination of mincemeat in Baghdad markets with different invaders.

However, while this study has focused on the detection and/or enumeration of $Y$. enterocolitica and $Y$. enterocolitica-like bacteria at near consumer levels, effective action to reduce or eliminate the risks posed by this organism will involve diverse and coordinated actions at a number of stages of the food chain. These include the incorporation and consistent application of Good Agricultural practice (GAP), Good Manufacturing practice (GMP), and Hazard Analysis of Critical Control Points (HACCP) at every stage of the beef supply chain, from the farm, through the abattoir, to the retailer, and those involved with the handling and processing of such raw meat products in the home environment. In addition, suitable intervention measures may be necessary to eliminate the pathogen in food reaching the consumer $(1,6$, $8,10,12 \& 13)$.

\section{References}

[1] Siriken, B. (2004). The Presence of Yersinia enterocolitica and Other Yersinia Species in Ground Beef in AydÝn, Turkey. Turk J Vet Anim. Sci.28: 489495.

[2] Stern, N.J., Pierson, M.D.: Yersinia enterocolitica: a review of the psychrotrophic water and foodborne pathogen. J. Food Sci., 1979; 47: 582-584, 588.

[3] Doyle, M.P., Cliver, D.O.: Yersinia enterocolitica. In: Foodborne Diseases. Ed. Cliver, D.O., Academic Press. Inc. Washington, 1990; 102-120.

[4] Kapperud, G.: Yersinia enterocolitica in food hygiene. Int. J. Food Microbiol., 1991; 12: 53-66.

[5] Ravagnan, G., Chiesa, C.: Yersiniosis: present and future. (1995). $6^{\text {th }}$ International Symposium Volume on Yersinia. Contrib. Microbiol. Immunol., 13: 340.

[6] Guven, A.; Sezer, C.; Aydin, B. D.; Oral, N. B. and Vatansever, L. (2010). Incidence and Pathogenicity of Yersinia enterocolitica Isolates from Foods in Turkey. Kafkas Univ Vet Fak Derg.16: S107-12.

[7] Zadernowska, A.; Chaje, W.; Wierzchowska, C. and Trokenheim, L. L. (2014). Yersiniaenterocolitica: A Dangerous, But Often Ignored, Foodborne Pathogen. Food Reviews International, 30:53-70.

[8] Sabina, Y.; Rahman, A.; Ray, R.C.; Montet, D. Yersinia enterocolitica; mode of transmission, molecular insights of virulence, and pathogenesis of infection. J. Pathog. 2011, 2011, 429069.

[9] Drummond, N.; Murphy, B. P. and Ringwood, T. (2012). Yersinia enterocolitica: a brief review of the issues relating to the zoonotic pathogen, public health challenges, and the pork production chain. Foodborne Pathog. Dis. 9:179-189.

[10]Morris, G. K. and Feeley, J. C. (1976). Yersinia enterocolitica: a review of its role in food hygiene. Bull. World Health Organ., 54: 79-85.

[11] Kapperud, G. (1991). Yersinia enterocolitica in food hygiene. Int. J. Food Microbiol.;12(1):53-65.

[12]EFSA.(2007a). Scientific Opinion of the Panel on BIOHAZ on a request from EFSA on monitoring and identification of human enteropathogenic Yersiniaspp. The EFSA Journal 595:1-30.

[13]EFSA.(2007b). Scientific Opinion of the Panel on Biological Hazards and Animal Health Animal Welfare on a request from the European Food Safety Authority (self-mandate) to issue a Scientific Opinion on the Review of the Community Summary Report on Trends and Sources of Zoonoses, Zoonotic Agents and Antimicrobial Resistance in the European Union in 2005, The EFSA Journal 600:1-32.

[14] Johnson, J. L. (1998). Chapter 9: Isolation and identification of pathogenic Yersinia enterocolitica from meat and poultry products. USDA/FSIS Microbiology Laboratory Guidebook ( ${ }^{\text {rd }}$ eds. $) ; 1-28$.

[15] ISO.(2003). Microbiology of food and animal feeding stuffs -Horizontal method for the detection of presumptive pathogenic Yersinia enterocolitica. ISO 10273-2003.

[16]Food and Drug Administrator. (2015).Yersinia enterocolitica. Bacteriological Analytical Manual Online. Weagant SD, Feng $\mathrm{P}$ editors. United States: 


\section{International Journal of Science and Research (IJSR) \\ ISSN (Online): 2319-7064}

Index Copernicus Value (2013): 6.14 | Impact Factor (2015): 6.391

Food and Drug Administration Center for Food Safety and Applied Nutrition.

[17] Wauters, G.; Kandolo, K. and Janssens, M. (1987). Revised biogrouping scheme of Yersiniaenterocolitica. Contr Microbiol Immunol, 9, 13-21.

[18] Schiemann, D. A. and Wauters, G.(1992). Yersinia. In, Vanderzant C, Splittstoesser F (Eds): Compendium of Methods for the Microbiological Examination of Foods, $3^{\text {rd }}$ Washington, DC, American Public Health Association, pp. 433-450.

[19] Laird, W. ænd Cavanough, D. C. (1980). Correlation of autoagglutination and virulence of Yersinia. J Clin Microbiol, 11, 430-432.

[20]Farmer, J. J.; Carter, G. P.; Miller, V. L.; Falkow, S. and Wachmuth, I. K. (1992). Pyrazinamidase, CR-MOX agar, salicin fermentation-esculin hydrolysis, and Dxylose fermentation for identifying pathogenic serotypes of Yersinia enterocolitica. J Clin Microbiol, 30, 25892594.

[21] Quinn, P.J.; Carter, M.E.; Markey, B. and Carter, G.R. (2004). Clinical Veterinary Microbiology. $2^{\text {nd }}$ ed., Mosby Int., USA.

[22] Jay, J.M.; Loessner, M.J. and Golden, D.A. (2005). Modern Food Microbiology, $7^{\text {th }}$ ed., Aspen Pub., Gathersburg, MD, USA.

[23] Miles, A. A.; Misra, S. S. and Irwin, J. O. (1938). The estimation of the bactericidal power of blood. J. hyg. Camp, 38: 739-749.

[24] Oxoid-Remel (2015). Biochemical panel and Serological test kits.

[25] SPSS. (2015). Statistical Package for the Social Sciences, Version 23. Chicago III, USA. Website http://www.spss.com. 